\title{
COVID-19 and Agricultural Workers: A Descriptive Study
}

\author{
Roxana Chicas $^{1}\left(\right.$ ) Nezahualcoyotl Xiuhtecutli ${ }^{2,3} \cdot$ Madelyn Houser $^{4} \cdot$ Sanne Glastra $^{5} \cdot$ Lisa Elon $^{6} \cdot$ Jeff M. Sands ${ }^{1}$. \\ Linda McCauley ${ }^{4} \cdot$ Vicki Hertzberg ${ }^{4}$
}

Accepted: 28 September 2021 / Published online: 12 October 2021

(C) The Author(s), under exclusive licence to Springer Science+Business Media, LLC, part of Springer Nature 2021

\begin{abstract}
Agricultural workers, designated as "essential" at the start of the COVID-19 pandemic, work in harsh labor conditions, and now have the added challenge of continuing to work during the COVID-19 pandemic. The aim of this study was to assess agricultural workers' COVID-19 related history, employer-based safety measures, individual preventive practices, and COVID-19 vaccination uptake. A questionnaire study was conducted among agricultural workers in Central Florida about COVID-19 during the month of June 2020 and again in July 2021. Among 92 agricultural workers in June 2020, 47\% were obese; $11 \%$ had had a COVID-19 nasal test; $87 \%$ were able to social distance at work and $34 \%$ reported employer provided face masks; $15 \%$ reported not willing to get the COVID-19 vaccine and 25\% were unsure. $40 \%$ could self-isolate if they contracted COVID-19. In a follow-up visit in July 2021, 53\% of participants reported receiving a COVID-19 vaccine. Agricultural workers are particularly vulnerable to COVID-19 due to existing health risk factors and lack of essential protective resources. Occupational health protections social safety net programs are urgently needed to prevent infections in vulnerable workers, and reduce community spread, and increase COVID-19 vaccination rates.
\end{abstract}

Keywords COVID-19 · Agricultural workers · Health behavior · Prevention and control

\section{Introduction}

The rapid spread of severe acute respiratory syndromecoronavirus 2 (SARS-CoV-2), the virus that causes coronavirus disease 2019 (COVID-19), has become a global public health crisis. In response to the highly contagious

Roxana Chicas

rchicas@emory.edu

1 Division of Renal Medicine, Department of Medicine, Emory University, 100 Woodruff Circle, Atlanta, GA 30322, USA

2 Anthropology Department, Tulane University, New Orleans, LA, USA

3 Farmworker Association of Florida, Apopka, FL, USA

4 Nell Hodgson Woodruff School of Nursing, Emory University, Atlanta, GA, USA

5 Department of Quantitative Theory and Methods, Emory College of Arts and Science, Emory University, Atlanta, GA, USA

6 Department of Biostatistics and Bioinformatics, Rollins School of Public Health, Emory University, Atlanta, GA, USA virus, shelter-in-place mandates and social distancing guidelines were issued throughout the United States beginning in March 2020. However, even with these guidelines, agricultural workers, designated as "essential" at the start of the pandemic [1], continue to work and are at increased risk for contracting COVID-19. Agricultural workers are primarily (75\%) composed of Hispanic immigrants from Mexico and Central America [2]. In the United States, Hispanics have been disproportionately affected by COVID-19, experiencing higher rates and more severe infection and increased mortality compared to non-Hispanics [3-5]. To date, there is no systematic tracking of positive COVID-19 cases by occupational sectors [6]; however, as of August 10, 2021, it is estimated that more than 620,000 agricultural workers have tested positive for COVID-19 nationwide, and over 22,000 have tested positive in Florida [7].

The nature of agricultural jobs puts workers at higher risk for infection as they work in close proximity to each other, for example while harvesting plants, working in packing plants, or riding in buses or vans to picking sites. While individuals may wear scarves or bandanas over their noses and mouths, their supervisors rarely offer personal protective equipment (PPE) $[8,9]$. The work is often physically 
demanding, and as a result, may induce heavy breathing, increasing the ease of transmission of respiratory viruses like COVID-19. Workers have limited access to water, soap, and utensils to help them maintain hand hygiene, and almost universally lack paid sick leave and health insurance $[10,11]$. Adding to the risks that agricultural workers face because of their occupation are such structural vulnerabilities as language barriers, foreign-born non-citizen status, poverty, low education [2, 12], and living in crowded, substandard housing conditions and multi-generational households [13, 14]. Agricultural workers are often hesitant to seek medical care due to the chilling effect of the 2019 Public Charge Rule that denies adjustment of immigration status to immigrants if they are deemed likely to receive public benefits [15] and ineligibility for the federal stimulus money sent to families through the coronavirus aid, relief, and economic security (CARES) Act [16]. Furthermore, agricultural workers have a high prevalence of chronic underlying medical conditions such as hypertension, diabetes, and overweight/obesity, which increase the risk for severe illness from COVID-19 [17]. The myriad of barriers has made it challenging for agricultural workers to protect themselves and their families from exposure to COVID-19 and has further exacerbated social inequities in an already marginalized group.

Our aim in this study was to assess agricultural workers' COVID-19-related history, employer-based safety measures implemented at the workplace, individual preventive practices, and COVID-19 vaccine uptake. Such information may inform interventions to mitigate the spread of COVID-19 among vulnerable populations such as agricultural workers and increase uptake of the COVID-19 vaccines.

\section{Methods}

In collaboration with the Farmworker Association of Florida (FWAF), in June 2020 we enrolled 92 participants from our longitudinal R01 study (OHEaRD-1R01OH011782-01) that is examining heat stress and kidney injury in Florida agricultural workers. Participants were 18-49 years of age and working in the agricultural sector for a minimum of 4 weeks prior to parent study enrollment. Parent study exclusion criteria included being pregnant or self-report of previous diagnosis with hypertension, diabetes mellitus type 1 or 2, glomerulonephritis, pyelonephritis, renal calculi, or snake bite. The Institutional Review Board at Emory University provided approval (IRB00112681) for this study.

FWAF staff contacted OHEaRD study participants, renewed consent, and administered an oral questionnaire. Data was recorded in Emory University's Research Electronic Data Capture (REDCap) [18, 19] system. Risk of COVID-19 exposure was reduced by providing face masks, hand sanitizers, marked walking paths, staggered appointments, and by maintaining physical distancing. Research staff wore appropriate PPE and sanitized the area before and after every participant.

The questionnaire included queries on diagnosis and testing for COVID-19, acceptability of vaccines, economic impact of COVID-19, employer-based COVID-19 safety measures implemented at the workplace, risk perception of COVID-19, and individual preventive actions taken to reduce COVID-19 exposure. We added additional questions midway through the study to assess emerging issues including reasons for not getting a COVID-19 test, concerns about a potential COVID-19 vaccine, history of getting the flu vaccine previously, and ability to stop work or to quarantine if exposed to or ill with COVID-19 $(n=39)$. Demographic and health information was obtained during a previous study visit (Jan-Feb, 2020). Body Mass Index (BMI) was calculated from measured height and weight. Hemoglobin A1c (HbA1c) was measured by the portable HbA1c detection instrument A1CNow + ${ }^{\circledR}$ (Bayer Diabetes Care, Sunnyvale, CA), and categorized as normal $(<5.7 \%)$, pre-diabetic $(5.7-6.4 \%)$, diabetic ( $\geq 6.5 \%)$. Systolic and diastolic blood pressure (SBP, DBP) were measured once after the participant had been seated for a minimum of five minutes using a digital system (Omron BP760N, Omron Healthcare Inc, Bannockburn, IL) and categorized as normal (SBP $<120$ and DBP $<80$ ), elevated (SBP 120-139 or DBP 80-89), and high (SBP $\geq 140$ or DBP $\geq 90$ ). Since the parent study is a longitudinal study, $\mathrm{HbA} 1 \mathrm{c}$ and blood pressure were measured to monitor changes over the workday and over 2 years.

In a follow-up parent study visit in July 2021, participants were asked if they had received the COVID-19 vaccine. Participants who reported they had not received the COVID-19 vaccine were asked the reason for not getting the vaccine.

The data is summarized using medians and quartiles and frequencies and percentages. All analyses used SAS v 9.4 software (SAS Institute, Cary, NC, USA).

\section{Results}

We enrolled 92 Hispanic agricultural workers with a median age of 40 (first and third quartile [Q1Q3]: 35, 45) (Table 1). The majority were female (77\%), and three-quarters were in a domestic partnership. Median years of education was 9 (Q1Q3: 6, 11), and all the participants identified as Hispanic, primarily from Mexico. Ninety-one percent reported they were currently working, with $36 \%$ working in ferneries and $50 \%$ in nurseries. The majority of the workers were either overweight $(36 \%)$ or obese $(47 \%) ; 11 \%$ had prediabetes HbA1c levels, and 3\% had a diabetes HbA1c level. Half of the participants had either elevated (37\%) or high (13\%) blood pressure measurements. 
Table 1 Socio-demographic and health characteristics of agricultural workers in the COVID-19 Study; Apopka and Pierson, Florida

\begin{tabular}{ll}
\hline Characteristics & Overall (n=92) \\
& Median [q1, q3], or \%, (n) \\
\hline Socio-demographic ${ }^{\mathrm{a}}$ & \\
Age (years) & $40[35,45]$ \\
Female & $77 \%(71)$ \\
Marital status, coupled & $74 \%(67)$ \\
Education (years) & $9[6,10]$ \\
Hispanic & $100 \%(91)$ \\
Nationality & \\
Mexico & $77 \%(71)$ \\
Central American & $16 \%(15)$ \\
United States & $3 \%(3)$ \\
Other & $3 \%(3)$ \\
Health & \\
Body mass index categories & \\
Normal (18.5-<25) & \\
Overweight (25-<30) & $18 \%(16)$ \\
Obese ( $\geq 30)$ & $36 \%(32)$ \\
A1c & $47 \%(42)$ \\
Normal &
\end{tabular}

${ }^{\text {a }}$ Collected in January2020 at OHEARD study initiation

${ }^{\mathrm{b}}$ Missingness: Hispanic $=1, \mathrm{~A} 1 \mathrm{c}=3, \mathrm{BMI}=2$

${ }^{\mathrm{c}}$ Collected in June2020 at special COVID-19 visit

When queried in June 2020, one agricultural worker out of 92 reported having had COVID-19, which was diagnosed with a COVID-19 nasal swab test (Table 2). Ten workers reported having a COVID-19 nasal swab test; half of these reported COVID-19 related symptoms. The primary reason given for not testing for COVID-19 was that they did not have COVID-19 symptoms (62\%, 24/39). Among all participants, $23 \%$ reported 1 or more symptoms that occur with COVID-19 infection: congestion or runny nose (17\%), fatigue (15\%), body aches (11\%), fever (7\%), cough (7\%), difficulty breathing (7\%), loss of taste or smell (7\%), sore throat (7\%), chills (7\%), and chest congestion (5\%). Only
Table 2 COVID-19 health characteristics and preventive practices of agricultural workers

\begin{tabular}{|c|c|}
\hline \multicolumn{2}{|l|}{ Characteristic } \\
\hline Personal & $\%,(\mathrm{n})$ \\
\hline \multicolumn{2}{|l|}{ History Of COVID-19 } \\
\hline Yes & $1 \%,(1)$ \\
\hline Don't know/not sure & $14 \%(13)$ \\
\hline \multicolumn{2}{|l|}{ Had A COVID-19 nasal test } \\
\hline Yes & $11 \%(10)$ \\
\hline If Yes, positive Covid-19 nasal test & $10 \%(1)$ \\
\hline \multicolumn{2}{|l|}{ Reasons for not getting COVID-19 test ${ }^{\mathrm{a}}$} \\
\hline No Covid-19 symptoms & $62 \%(24)$ \\
\hline Do not think exposed to COVID-19 & $8 \%(3)$ \\
\hline Cannot miss work & $8 \%(3)$ \\
\hline \multicolumn{2}{|l|}{ Willing to get COVID-19 vaccine } \\
\hline Yes & $60 \%(55)$ \\
\hline No & $15 \%(14)$ \\
\hline Don't know/not sure & $25 \%(23)$ \\
\hline \multicolumn{2}{|l|}{ COVID-19 vaccine concerns ${ }^{\mathrm{b}}$} \\
\hline Does not trust COVID-19 vaccines & $35 \%(13)$ \\
\hline Usually get the flu vaccine $^{\mathrm{a}}$ & $59 \%(23)$ \\
\hline \multicolumn{2}{|l|}{ Individual Covid-19 preventive practices } \\
\hline Wash or disinfect hands frequently & $98 \%(90)$ \\
\hline Avoid large crowds & $98 \%(90)$ \\
\hline Adhered to local Government guidelines & $96 \%(88)$ \\
\hline Maintain 6 feet distance from others & $95 \%(87)$ \\
\hline Disinfecting surfaces & $93 \%(86)$ \\
\hline Wears facemasks & $84 \%(77)$ \\
\hline Stop going to work & $39 \%(36)$ \\
\hline Eligible for the CARES act relief funds & $18 \%(17)$ \\
\hline \multicolumn{2}{|l|}{ Workplace } \\
\hline Employer provided relief funds & $17 \%(16)$ \\
\hline \multicolumn{2}{|l|}{ Workplace Covid-19 preventive practices } \\
\hline Maintain social distancing ( $2 \mathrm{~m}$ between workers) & $87 \%(79)$ \\
\hline Employer provides facemasks & $34 \%(31)$ \\
\hline Carpool to work & $16 \%(15)$ \\
\hline Employer takes temperature at start of shift & $12 \%(11)$ \\
\hline \multicolumn{2}{|l|}{ Perceived COVID-19 risk at workplace } \\
\hline High risk & $2 \%(2)$ \\
\hline Medium risk & $32 \%(29)$ \\
\hline Low risk & $36 \%(33)$ \\
\hline No risk & $26 \%(24)$ \\
\hline Don't know/not sure & $4 \%(4)$ \\
\hline Lost work during COVID-19 outbreak & $75 \%(69)$ \\
\hline
\end{tabular}

Apopka and Pierson, Florida; June 2020. N=92

${ }^{a}$ Asked only at one study location, $\mathrm{n}=39$

bConcerns among those who said "no/don't know/not sure" 
$40 \%$ said they would be able to self-isolate if they had COVID-19.

Asked if they would be willing to get a COVID-19 vaccine when it becomes available, $60 \%$ answered yes, $15 \%$ answered no, and $25 \%$ were unsure. Positive attitudes about getting the vaccine for COVID-19 paralleled attitudes about getting the flu vaccine, with 59\% reporting that they do usually get the flu vaccine. Seventy percent of those usually getting the flu vaccine said they would be willing to vaccinate against COVID-19, compared with 57\% saying unsure or no. The main concern for those who were unsure or unwilling to get the COVID-19 vaccine when available was that they would not trust COVID-19 vaccines (33\%).

We also assessed preventive actions participants were taking to reduce exposure to COVID-19. Outside of the workplace, over $90 \%$ frequently washed their hands or used hand sanitizer, avoided large social gatherings, or eating at restaurants, and disinfected surfaces. Eighty-four percent used facemasks.

While $91 \%$ of participants reported they were currently working, 75\% had lost work hours or were furloughed. Only $18 \%$ reported eligibility for the CARES Act relief funds, and $17 \%$ reported receiving employer-provided relief funds.

Employer-enabled COVID-19 safety measures included maintaining at least 6 feet between workers $(87 \%)$, provision of facemasks (34\%), and pre-shift temperature checks (12\%). Sixteen percent carpooled to work. When asked about their perceived risk of contracting COVID-19 at work, 74\% felt there was some risk (2\% high, 32\% medium, $36 \%$ low, and $26 \%$ no risk). There was no significant association between perceived workplace risk and employer-initiated safety measures (masking, social distancing, temperature taking).

In July 2021, we followed-up with 81 of the 92 COVID19 sub-study participants. Forty-eight percent reported complete and 5\% reported partial COVID-19 vaccination (Table 3). Of those in June 2020 willing to get the COVID19 vaccine once it became available, $52 \%$ reported receiving at least one dose. Among the originally unwilling, 67\% received at least one dose, compared to $56 \%$ unsure, and $53 \%$ of the willing. Fifteen percent would not get the vaccine because "you get sick after getting the shot." Mistrust in the COVID-19 vaccine was reported by $11 \%$, and $5 \%$ reported they did not have time to be vaccinated. The Moderna vaccine was the most common (43\%), followed by Johnson \& Johnson's (31\%) and Pfizer-BioNTech's (26\%).

\section{Discussion}

We evaluated COVID-19-related practices and its impact on 92 Hispanic agricultural workers in Central Florida and found major health and work risk factors were common among these workers. Workers took personal preventive
Table 3 Uptake of COVID-19 vaccine by agricultural workers

\begin{tabular}{ll}
\hline COVID-19 vaccine query & $\%,(\mathrm{n})$ \\
\hline Received COVID-19 vaccine & \\
Yes & $53 \%,(42)$ \\
No & $48 \%(38)$ \\
If yes, COVID-19 vaccine type and completion status & \\
Johnson \& Johnson single-dose vaccine & $31 \%(13)$ \\
Moderna, two doses & $38 \%(16)$ \\
Pfizer-BioNTech, two doses & $21 \%(9)$ \\
1 dose of Moderna or Pfizer-BioNTech & $10 \%(4)$ \\
Reason for not getting COVID-19 vaccine & \\
Side effects: "you get sick after getting the shot” & $32 \%(12)$ \\
Mistrust in COVID-19 vaccine & $26 \%(10)$ \\
No time to get the COVID-19 vaccine & $11 \%(4)$ \\
Not necessary & $11 \%(4)$ \\
Other & $21 \%(8)$ \\
\hline
\end{tabular}

Apopka and Pierson, Florida; July 2021. N=81

precautions seriously, but workplace preventions were uncommon. This is congruent with reports of disproportionately greater impact of COVID-19 among Hispanics [3], and news media reports of large outbreaks among agricultural workers in Florida [20-22].

Obesity (body mass index (BMI) $\geq 30$ ), elevated blood pressure, and prediabetes are risk factors for contracting COVID-19 and are associated with severe COVID-19 illness and mortality [23-26]. Sixty-nine percent of our participants had at least one of those risk factors, and 37\% had multiple risk factors. This suggests that if agricultural workers do contract COVID-19, they might be more likely to experience adverse outcomes than the general population.

Despite the many infection risks faced by agricultural workers, only $11 \%$ reported getting COVID-19 testing, and one participant tested positive. One confirmed case among 92 similar adults is much lower than expected in a vulnerable, unprotected workforce. For comparison, in Florida in June 2020, the highest daily positive rate for COVID-19 was $17 \%$ [27]. Although participants reported symptoms that occur with COVID-19 infection, the main reason for not getting a COVID-19 nasal test was not having COVID19 symptoms. Anecdotally, participants considered the symptoms they experienced to be related to other factors. For instance, fatigue and body aches were attributed to the number of hours worked, and stuffy nose and sore throat to seasonal allergies. Thus, the number of workers who had COVID-19 is likely to be higher than 1 out of 92 because of lack of testing as well as asymptomatic infection.

Although the participants overwhelmingly reported individual practices of COVID-19 preventive measures such as using a face mask and social distancing, there was a lack of employer-based COVID-19 safety measures implemented 
at the workplaces. Only a third of the participants reported that their employer provided face masks, and an even a lower percentage reported having their temperature checked at the start of the work shift. All of the workers in our study were settled agricultural workers who lived in independent housing, often with family members. A growing proportion of the agricultural worker population in the U.S., however, are entering the country on H-2A visas. These workers, primarily young males, live in communal housing provided by their employers with shared sleeping environments, baths, and kitchens $[13,28]$. A recent article discussed the COVID-19 hazards associated with agricultural worker housing along with the inability to change the regulations in recognition of the pandemic [14]. Alarmingly, neither the agricultural industry nor the federal government have established a protection standard for occupational exposure to COVID-19 for essential workers, such as those in agriculture $[6,8,9]$. More than half of the workers in this study perceived no risk or low risk of COVID-19 exposure at the workplace; this perception may be attributed in part to the lack of enforced workplace protective measures by both the industry and government occupational agencies, such as the occupational safety and health administration (OSHA), CDC, and the United States Department of Agriculture (USDA). The practices needed to reduce infection risk among agricultural workers have been detailed, including the need for widespread testing of this population [29].

The labor markets have been disrupted by the COVID19-pandemic, leading to massive lay-offs, furloughs, and decreases in work hours, to the point that the United States government on March 27, 2020 passed the CARES Act to support families with relief funds. Despite categorization of agricultural workers as "essential workers," $75 \%$ of participants reported losing work (furlough or reduced work hours) since the start of the pandemic, and only $18 \%$ were eligible for CARES Act relief funds. About the same number of workers reported receiving employer-based relief funds.

Sixty percent of participants reported a willingness to get the COVID-19 vaccine, a quarter were unsure, and $15 \%$ were unwilling. COVID-19 vaccination in our cohort (53\%) was lower than in Florida's overall population (59\%) but higher than Florida's overall Hispanic population (33\%) [30]. In contrast, during the $2009 \mathrm{H} 1 \mathrm{~N} 1$ pandemic, a random sample drawn from the Knowledge Networks assessed the public willingness to take the H1N1 vaccine and found that $17 \%$ of Hispanics reported "yes," 47\% "no," and 36\% "don't know" [31]. The uptake of the H1N1 vaccine was lowest in foreign-born Hispanics (15\%) living in the U.S. compared to U.S. born Hispanics (18\%) and White non-Hispanics (21\%) [32]. The higher vaccine uptake in our cohort may be due to the devastating socio-economic impact (lockdowns, school becoming virtual, jobs lost, etc.) that COVID19 has had and the disproportionately higher COVID-19 cases, hospitalizations, and deaths among Hispanics [3-5]. However, the relatively low vaccination rate among our farmworkers is concerning, especially considering the rising COVID-19 variant cases [33] and the sharp decline in COVID-19 vaccination rates nationwide since mid-April 2021 [34]. In addition, a study using death records from the California Department of Public Health found that food/ agriculture workers had a 39\% mortality increase (risk ratio $\mathrm{RR}=1.39 ; 95 \% \mathrm{CI} 1.32-1.48)$ relative to the pre-pandemic period [35].

Concern about side effects was the primary reason for not getting the COVID-19 vaccine. COVID-19 vaccines may produce side effects such as tiredness, muscle pain, fever, and headaches which can affect daily activities for a few days [36]. This could interfere with agricultural workers' ability to perform the physically demanding tasks that their occupation requires. Additionally, most agricultural workers have low wages and no paid sick leave or paid vacation [37]; thus, agricultural workers may be avoiding the risk of taking leave to recover from the vaccine. Exposure to misinformation may also be distorting perceptions of postvaccine side effects. The second most common reason for not getting the COVID-19 vaccine was mistrust in the vaccine. While mistrust may be due to the novelty of COVID-19 and its treatments and the rapidity of vaccine development, it could also suggest that the politicization of the COVID-19 outbreak response and vaccine development is contributing to a mistrust of public health information in this vulnerable population [38].

Limitations of this study include that it was a convenience sample of Florida agricultural workers that may not be generalizable to the wider Florida or U.S. population of agricultural workers. Additionally, all data were self-reported and dependent on the participants' honesty and memory. While the COVID-19 questionnaire was developed with our community partner, the FWAF, to ensure it was culturally appropriate and applicable to the agricultural population, it has not been validated. Despite these limitations, our findings provide valuable information about the health and work risk factors for COVID-19 in agricultural workers.

\section{Recommendations}

During this study, few participants had been tested, with most of them citing absence of symptoms as their primary reason for not doing so. Information about transmission of the virus by asymptomatic individuals needs to be repeatedly made in public health messages targeted to the farmworker population. The spread of the variant strains by vaccinated persons with little or no symptoms has demonstrated the importance of this communication. Worksite testing or employer partnership with local clinics or community 
organizations to conduct regularly scheduled COVID-19 rapid antigen diagnostic immunoassay (RAD) tests may identify workers early enough to contain and stop further transmission of COVID-19 amongst co-workers and communities. A field-based study showed that RAD performed well as a point-of-care test [39].

Increasing the COVID-19 vaccination rate in the agricultural population is critical to reducing the transmission of SARS-CoV-2 and resuming pre-COVID-19 activities. Community campaigns targeted to agricultural workers should include education on normal COVID-19 vaccine side effects and their relationship to building immunity, as well as addressing misinformation about adverse side effects. The availability of worksite vaccination would address vaccine hesitancy due to lack of time, transportation, or fear of contact with public officials. Furthermore, agricultural workers generally have low wages and no paid sick time if they have post-vaccine symptoms that interfere with their ability to work; thus, a social safety net program, such as providing a financial incentive for the uptake of COVID-19 vaccines is necessary to protect against lost wages from post-COVID-19 vaccine side effects.

\section{Conclusion}

During the COVID-19 pandemic, "essential workers" such as agricultural workers are at increased risk for contracting COVID-19. Agricultural workers are particularly vulnerable to COVID-19 due to existing health risk factors and lack of essential protective resources. Federal worksite prevention and control standards are needed to protect agricultural workers and reduce community spread of COVID-19. These standards should include provision of PPE, regular access to COVID-19 testing, as well as educational material about community spread and the need to take preventive measures regardless of the presence of symptoms or vaccination status. Targeted community campaigns to increase COVID19 vaccine rates are essential. Such measures are critical to protect the workers, their families, and the communities in which they live and to ensure availability of workers in an essential industry.

Funding This work was supported by the National Institute of Diabetes and Digestive and Kidney Diseases (NIDDK) T32-DK 07656HHS and National Institute for Occupational Safety and Health (NIOSH) 1R01OH011782.

\section{References}

1. CISA. Advisory memorandum on ensuring essential critical infrastructure workers ability to work during the COVID-19 response. Washington, DC: Cybersecurity \& Infrastructure Security Agency; 2020.

2. Hernandez T, Gabbard S. Findings from the National Agricultural Workers Survey (NAWS) 2015-2016: a demographic and employment profile of United States farmworkers. Washington, DC: Department of Labor, Employment and Training Administration; 2019. p. 84.

3. US Centers for Disease Control and Prevention. COVID-19 in Racial and Ethnic Minority Groups (2020). https://www.cdc. gov/coronavirus/2019-ncov/need-extra-precautions/racial-ethnic-minorities.html. Accessed 18 Jul 2020.

4. Hooper MW, Nápoles AM, Pérez-Stable EJ. COVID-19 and racial/ethnic disparities. JAMA. 2020;323:2466.

5. Tian T, Zhang J, Hu L, Jiang Y, Duan C, Wang X, et al. Risk factors associated with mortality of COVID-19 in 2692 counties of the United States. medRxiv. 2020;65:101.

6. Hanage WP, Testa C, Chen JT, Davis L, Pechter E, Seminario $P$, et al. COVID-19: US federal accountability for entry, spread, and inequities-lessons for the future. Eur J Epidemiol. 2020;35:995.

7. Purdue Food and Agricultural Vulnerability Index Purdue University (2019). https://ag.purdue.edu/agecon/Pages/FoodandAgV ulnerabilityIndex.aspx?_ga $=2.49471334 .1159720487 .16001$ 11458-250602208.1598985334. Accessed 10 Aug 2021.

8. Coleman ML. Essential Workers Are Being Treated As Expendable. The Atlantic (2020).

9. Campbell M. Farmworker are getting coronavirus. They face retaliation for demanding safe conditions. The World (2020). https:// www.pri.org/stories/2020-07-29/sick-covid-19-farmworkers-faceretaliation-demanding-safe-conditions. Accessed 10 Aug 2020.

10. Guild A, Figueroa I. The neighbors who feed us: farmworkers and government policy-challenges and solutions. Harvard Law Policy Rev. 2018;13:157.

11. Farmworker Justice. Statement by Farmworker Advocates on COVID-19 and the Risks to Farmworkers. Farmworker Justice (2020). https://www.farmworkerjustice.org/blogpost/statementby-farmworker-advocates-on-covid-19-and-the-risks-to-farmw orkers/. Accessed 23 Nov 2020.

12. Boggess B, Bogue HO. The health of U.S. agricultural worker families: a descriptive study of over 790,000 migratory and seasonal agricultural workers and dependents. J Health Care Poor Underserved. 2016;27(2):778-92.

13. Moss Joyner A, George L, Hall ML, Jacobs IJ, Kissam ED, Latin $S$, et al. Federal farmworker housing standards and regulations, their promise and limitations, and implications for farmworker health. New Solut. 2015;25(3):334-52.

14. Accorsi EK, Samples J, McCauley LA, Shadbeh N. Sleeping within six feet: challenging Oregon's Labor Housing COVID-19 guidelines. J Agromed. 2020;25:413.

15. The L. US public charge rule: pushing the door closed. Lancet (London, England). 2019;393(10187):2176.

16. Wilson FA, Stimpson JP. US policies increase vulnerability of immigrant communities to the COVID-19 pandemic. Ann Glob Health. 2020;86(1):57.

17. CDC. Coronavirus Disease 2019 (COVID-19) People with Certain Medical Conditions: Centers for Disease Control and Prevention; (2020). https://www.cdc.gov/coronavirus/2019-ncov/need-extraprecautions/people-with-medical-conditions.html. Accessed 29 Oct 2020.

18. Harris PA, Taylor R, Thielke R, Payne J, Gonzalez N, Conde JG. Research electronic data capture (REDCap)-a metadatadriven methodology and workflow process for providing translational research informatics support. J Biomed Inform. 2009;42(2):377-81.

19. Harris PA, Taylor R, Minor BL, Elliott V, Fernandez M, O’Neal $\mathrm{L}$, et al. The REDCap consortium: building an international 
community of software platform partners. J Biomed Inform. 2019;95:103208.

20. Gomez-Licon A. Florida migrant towns become coronavirus hot spots in US. New York: ABC News; 2020.

21. Mazzei P. Florida's coronavirus spike is ravaging migrant farmworkers. New York: New York Times; 2020.

22. Grinspan L. 'I really thought I was going to die.' Plant nursery workers sick with COVID-19 report dangerous conditions at work. Doral: Miami Herald; 2020.

23. US Centers for Disease Control and Prevention. Evidence used to update the list of underlying medical conditions that increase a person's risk of severe illness from COVID-19 https://www. cdc.gov/coronavirus/2019-ncov/need-extra-precautions/evidencetable.html. Accessed 18 Jul 2020.

24. Smith SM, Boppana A, Traupman JA, Unson E, Maddock DA, Chao K, et al. Impaired glucose metabolism in patients with diabetes, prediabetes, and obesity is associated with severe COVID19. J Med Virol. 2021;93:409.

25. Yang J, Zheng Y, Gou X, Pu K, Chen Z, Guo Q, et al. Prevalence of comorbidities and its effects in patients infected with SARSCoV-2: a systematic review and meta-analysis. Int J Infect Dis IJID. 2020;94:91-5.

26. Hussain A, Mahawar K, Xia Z, Yang W, El-Hasani S. Obesity and mortality of COVID-19. Meta-analysis. Obes Res Clin Pract. 2020;14(4):295-300.

27. Jankowski J. UPDATE: track Florida's rate of positive COVID19 tests (2020). https://covidactnow.org/us/florida-fl?s=1408382. Accessed 8 Dec 2020.

28. Quandt SA, Brooke C, Fagan K, Howe A, Thornburg TK, McCurdy SA. Farmworker housing in the United States and its impact on health. New Solut. 2015;25(3):263-86.

29. Tutor Marcom R, Freeman Lambar E, Rodman B, Thomas G, Watson A, Parrish B, et al. Working along the Continuum: North Carolina's collaborative response to COVID-19 for migrant \& seasonal farmworkers. J Agromed. 2020;25:409.

30. USA Facts. Florida Coronavirus Vaccination Progress (2021). https://usafacts.org/visualizations/covid-vaccine-tracker-states/ state/florida. Accessed 10 Aug 2021.

31. Quinn SC, Kumar S, Freimuth VS, Kidwell K, Musa D. Public willingness to take a vaccine or drug under emergency use authorization during the $2009 \mathrm{H} 1 \mathrm{~N} 1$ pandemic. Biosecur Bioterror. 2009;7(3):275-90.

32. Burger AE, Reither EN, Hofmann ET, Mamelund S-E. The influence of hispanic ethnicity and nativity status on 2009 H1N1 pandemic vaccination uptake in the United States. J Immigr Minor Health. 2018;20(3):561-8.

33. Rattner N, Mendez R. Covid cases are rising again in all 50 states across U.S. as delta variant tightens its grip. Englewood Cliffs: CNBC; 2021.

34. CDC. Trends in Number of COVID-19 Vaccinations in the US: Centers for Disease Control and Prevention; (2021). https://covid. cdc.gov/covid-data-tracker/\#vaccination-trends. Accessed 10 Aug 2021.

35. Chen YH, Glymour M, Riley A, Balmes J, Duchowny K, Harrison R, et al. Excess mortality associated with the COVID-19 pandemic among Californians 18-65 years of age, by occupational sector and occupation: march through November 2020. PLoS ONE. 2021;16(6):e0252454.

36. CDC. Possible side effects after getting a COVID-19 vaccine: Centers for disease control and prevention (2021) https://www. cdc.gov/coronavirus/2019-ncov/vaccines/expect/after.html. Accessed 10 Aug 2021.

37. US Department of Labor. Findings from the National Agricultural Workers Survey (NAWS) 2015-2016: a demographic and employment profile of United States farmworkers. Washington DC: US Department of Labor; 2018.

38. Halpern LW. The politicization of COVID-19. Am J Nurs. 2020;120(11):19-20.

39. Albert E, Torres I, Bueno F, Huntley D, Molla E, FernándezFuentes MÁ, et al. Field evaluation of a rapid antigen test (Panbio ${ }^{\text {TM }}$ COVID-19 Ag rapid test device) for COVID-19 diagnosis in primary healthcare centres. Clin Microbiol Infect. 2021;27(3):472.e7-.e10.

Publisher's Note Springer Nature remains neutral with regard to jurisdictional claims in published maps and institutional affiliations. 